\title{
The role of community pharmacy-based vaccination in the USA: current practice and future directions
}

This article was published in the following Dove Press journal:

Integrated Pharmacy Research and Practice

I July 2015

Number of times this article has been viewed

\author{
Albert T Bach \\ Jeffery A Goad \\ School of Pharmacy, Chapman \\ University, Irvine, California, USA
}

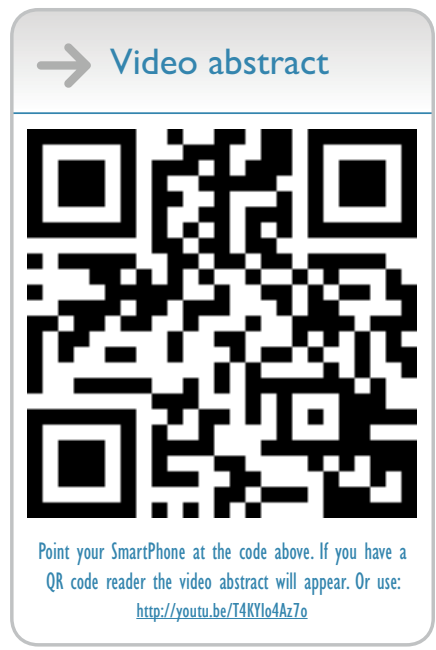

Correspondence: Albert Bach School of Pharmacy, Chapman University, 940I Jeronimo Rd, Irvine,

CA 92618 , USA

$\mathrm{Tel}+\mathrm{I} 7145165484$

$\mathrm{Fax}+\mid 714516548$

Email abach@chapman.edu

\begin{abstract}
Community pharmacy-based provision of immunizations in the USA has become commonplace in the last few decades, with success in increasing rates of immunizations. Community pharmacy-based vaccination services are provided by pharmacists educated in the practice of immunization delivery and provide a convenient and accessible option for receiving immunizations. The pharmacist's role in immunization practice has been described as serving in the roles of educator, facilitator, and immunizer. With a majority of pharmacist-provided vaccinations occurring in the community pharmacy setting, there are many examples of community pharmacists serving in these immunization roles with successful outcomes. Different community pharmacies employ a number of different models and workflow practices that usually consist of a year-round in-house service staffed by their own immunizing pharmacist. Challenges that currently exist in this setting are variability in scopes of immunization practice for pharmacists across states, inconsistent reimbursement mechanisms, and barriers in technology. Many of these challenges can be alleviated by continual education; working with legislators, state boards of pharmacy, stakeholders, and payers to standardize laws; and reimbursement design. Other challenges that may need to be addressed are improvements in communication and continuity of care between community pharmacists and the patient centered medical home.
\end{abstract}

Keywords: immunization, pharmacy practice, pharmacists, continuity of care

\section{Background}

An estimated 40,000 to 50,000 adults die annually from vaccine-preventable disease in the USA. ${ }^{1}$ Treating these patients who go without routine and recommended vaccinations costs the health care system US $\$ 10$ billion each year. ${ }^{2}$ Adult vaccination rates for routine recommended vaccines in the USA are low, and well below projected Healthy People 2020 targets. ${ }^{3,4}$ An example of this is the annual influenza vaccination, which is recommended for all people $\geq 6$ months of age. The most recent Centers for Disease Control and Prevention (CDC) data, from the 2012-2013 influenza season, revealed an influenza immunization rate of $45 \%$ in all people 6 months of age and older. There is also drastic variability in coverage among different age groups. In adults 18 years and older, the influenza vaccination rate increased with increasing age - from $31.1 \%$ in those $18-49$ years of age to $66.2 \%$ in those $\geq 65$ years of age. ${ }^{5}$ Looking at other vaccines, the pneumococcal polysaccharide vaccination rate for high-risk patients aged 19-64 years of age was 20\%. The Advisory Committee on Immunization Practices recommends a single dose of zoster vaccine for all adults aged 60 years and older, whether or not they report a prior episode of herpes zoster. ${ }^{6}$ However, overall, the shingles vaccination rate is only $20.1 \%$. $^{3}$ These are just a few examples of the low immunization rates seen today in the USA. 
The underutilization of these widely available vaccines has created an opportunity for pharmacists to play a role in improving immunization rates and thus advancing public health. Community pharmacy-based vaccination services have increased the number of immunization providers and the number of sites where patients can receive immunizations. It is thus important to understand the current role of community pharmacy-based immunization programs in the USA to identify opportunities and challenges for improving the public-health infrastructure to deliver vaccinations.

\section{Objectives}

This review explores the roles of the pharmacist as educator, facilitator, and immunizer in community pharmacy-based vaccination services. The review also touches on the journey to pharmacist as providers of vaccinations, delivery models, barriers, and future directions in the area of pharmacy-based vaccination services.

\section{Pharmacists as providers of vaccinations}

With $93 \%$ of Americans living within 5 miles of a community pharmacy, community pharmacists are one of the most readily accessible health care professionals in America. ${ }^{7}$ In 2011-2012, 20.1\% of US adults received their influenza vaccinations from a supermarket or drug store - an increase from $18.4 \%$ during the $2010-2011$ season. ${ }^{89}$ With multiple locations in metropolitan and urban areas, extended hours, reduced cost to administer vaccines, ${ }^{10}$ and convenience, community pharmacies address many factors that can increase immunization rates. ${ }^{11,12}$ According to the US Bureau of Labor Statistics, there were 286,400 pharmacist jobs in $2012 .{ }^{13}$ An estimated $51 \%$ of these pharmacists work in the 39,000 chain and 23,117 independent community pharmacy locations across the USA., ${ }^{74}$ Patients often report that their preference for receiving vaccinations in a pharmacy or from a non-physician in a nontraditional setting is due to convenience. ${ }^{15-17}$ Most immunizing community pharmacies provide vaccinations without an appointment, require almost no waiting, and do not charge visit fees. Community pharmacy hours of operation also provide extended access outside of traditional settings, such as a physician's office, which has limited hours after $5 \mathrm{pm}$ and is usually closed on the weekends and holidays. When immunizations are offered through community pharmacies during these "off-hours", large numbers of patients take advantage of the increased access and convenience, especially those younger, healthier adults in whom rates of immunizations tend to be lower. ${ }^{18}$
In addition to capturing the younger, healthier population, community pharmacies have been able to reach medically underserved and difficult-to-reach populations. ${ }^{11,19} \mathrm{Commu}-$ nity pharmacies offering vaccinations serve to complement the health care system to increase the number of providers and settings from which patients can receive vaccines.

As the pharmacy profession continues to move away from strictly product distribution to direct patient care, so does the education and training of pharmacists. Since 2000, the doctor of pharmacy (Pharm D) degree has been the sole degree offered to pharmacy graduates in the USA. All professional programs that graduate students with the Pharm D undergo accreditation by the Accreditation Council for Pharmacy Education (ACPE), which has set standards for professional competencies and outcome expectations of Pharm D graduates. In the 2006 Accreditation Standards and Guidelines for the Professional Program in Pharmacy Leading to the Doctor of Pharmacy Degree (hereafter, "ACPE Standards"), standard number 12 notes that the professional pharmacist competencies that must be achieved by graduates through the Pharm D curriculum are the ability to: provide patient-centered care; provide population-based care; manage human, physical, medical, information, and technological resources; manage medication-use systems; and promote the availability of effective health and disease-prevention services and health policy. ${ }^{20}$ It is the emphasis on providing population-based care and disease-prevention services that has prepared pharmacists to provide public-health services, which would include efforts to increase immunization rates, reach at-risk populations, and decrease the incidences and mortality of vaccine-preventable diseases. The ACPE Standards 2016 has now explicitly listed under the public-health domain that "[providing] an avenue through which students become certified in immunization delivery" ${ }^{\prime 21}$ is a required element of any Pharm D curriculum. In addition to a curriculum that produces pharmacists capable of identifying and solving public-health issues, the Pharm D graduate's training and expertise lies with pharmaceuticals. Since vaccines and biological products are approved by the US Food and Drug Administration and considered prescription (pharmaceutical) products, pharmacists are uniquely qualified to not only serve as drug experts but also vaccine experts as well.

Pharmacy-based immunization training for pharmacists also follows a nationally adopted certificate training program developed by the American Pharmacists Association (APhA), and the quality and content of this program in preparing pharmacists to administer vaccines are recognized by the CDC's National Immunization Program. ${ }^{22}$ The APhA 
Pharmacy-Based Immunization Delivery program involves three components: 1) a 12-hour self-study that covers immunology, vaccines, patient care, practice implementation, and legal and regulatory issues; 2) a live 8-hour program that reinforces and expands on the self-study content and focuses on immunization decision-making and pharmacy-practice implementation; and 3) a practicum that assesses subcutaneous and intramuscular injection techniques. ${ }^{23}$ This program has trained more than 150,000, and counting, pharmacists and student pharmacists nationwide. ${ }^{22,24}$

With the public's increased ability to access pharmacists, pharmacists are perfectly positioned and have notable training to engage in conversations with patients to prompt vaccine reminders and recommendations, and dispel myths and misconceptions regarding vaccinations. ${ }^{25}$ In addition to this, pharmacists have consistently ranked as one of the top three most honest and ethical professions in the USA. ${ }^{26}$

\section{Journey to pharmacist-provided vaccinations in the USA}

Although community pharmacies and pharmacists are recognized today by the CDC and the US Food and Drug Administration as established nontraditional settings and providers, most of this achievement took place just within the last two decades. Pharmacy involvement with vaccines and immunization in the USA has been noted since the mid-1800s but was limited in function, with pharmacies serving as a source of storage, preparation, distribution, and education, and as hosts of immunization providers in their practice. ${ }^{22,27}$ In 1993, then-secretary of the US Department of Health and Human Services (DHHS) Donna Shalala requested the APhA to define the role of pharmacists in improving vaccination rates. The APhA's response was successful in getting the DHHS to officially recognize that pharmacists are able to play an integral role in vaccine education, mobilization, distribution, access, record keeping, administration, and, ultimately, improving vaccination rates. ${ }^{22}$ From this, the first organized immunization training program for pharmacist-administered immunizations took place in Seattle, Washington, in late 1994. Pharmacists' movement into adult immunization came in 1996 and resulted from collaboration between the Mississippi Pharmacists Association and the APhA to address the Mississippi Department of Public Health's request for pharmacists in resolving the disparities in adult influenza and pneumococcal rates. This effort lead to the development of the now nationally recognized APhA Pharmacy-Based Immunization Delivery certificate program. ${ }^{22}$
Turf wars with physicians continue to be a challenge as pharmacists look to expand the ages of patients they can vaccinate, the types of vaccines they can administer, and their responsibilities by becoming independent providers of immunizations. The American College of Physicians supports pharmacists as a source of immunization information, facilitators of immunizations, and as immunizers, as appropriate and allowed by state law. ${ }^{28}$ In March of 2014, the National Vaccine Advisory Committee published its recommendations for adult immunization standards..$^{29}$ These recommendations call for all providers, including pharmacists, to implement policies and processes that increase adult vaccination. Most mainstream medical and allied health care provider organizations have signed on to promote this document. While there has been widespread support of pharmacy-based immunization programs under collaborative practice agreements, physician resistance and attitudes tend to be less favorable when community pharmacists take on an independent role in patient care. ${ }^{30,31}$ These opinions may vary among states, as an example of support was the California Association of Physician Groups helping California pharmacists achieve provider status and independent immunization practice in 2013 under Senate Bill 493. ${ }^{32,33}$

\section{State-based pharmacist immunization laws}

In the USA, all health care is delivered based not on federal but state law. Currently, all 50 states in the USA, and Washington DC and Puerto Rico, allow pharmacists to administer vaccinations. However, among states, laws vary for the need for a protocol and/or prescription to give a vaccine, minimum age limit, and the vaccines pharmacists are authorized to administer. The latest APhA and National Alliance of State Pharmacy Associations' survey of state immunization laws/rules, report that 31 states require pharmacist-administered vaccines to be pursuant to a protocol or prescription, while 12 allow for a protocol, prescription, or no prescriber needed (depending on the age and/or vaccine). ${ }^{34}$ The remaining states and US territories allow pharmacists to administer vaccines via protocol only or prescription only. Regarding age and types of vaccines, 27 states do not have a patient age limitation for pharmacistadministered vaccines, and 46 states currently authorize pharmacists to administer any type of vaccine. Patient age limitations in states range from those 5 years of age or older to those greater than 18 years of age. State laws also vary in the authority for student pharmacists to administer vaccines (eight states do not allow student pharmacists to vaccinate). 
The State of California was the most recent state to move from a 1995 law that allowed pharmacists to "administer immunizations pursuant to a protocol with a prescriber" to independent initiation of vaccines for those 3 years of age and older. ${ }^{35}$ Information on current state laws pertaining to pharmacist authority to immunize is updated regularly on the APhA's Immunization Center webpage (http://www. pharmacist.com/immunization-center).

\section{Role of the pharmacist in immunizations}

In 1997, the APhA published a set of guidelines for pharmacybased immunization advocacy that can still be applied today to the role of community pharmacists in immunizations. ${ }^{36}$ The organization encourages all pharmacists to adopt a level of involvement in vaccinations as either an educator (encouraging immunizations to patients served), facilitator (serving as a depot for vaccine distribution or host for immunizers), or immunizer (administering vaccinations consistent with state law).$^{36}$ Today, community pharmacists still serve in vaccine advocacy roles within their practice setting.

Although this review focuses on the role of community pharmacists in providing vaccinations, it is worth noting that pharmacists also play an important role in immunizations in the inpatient and health-systems setting. In this setting, pharmacists primarily serve as educators and facilitators to increase immunization rates. As part of the health care team, pharmacists provide immunization screening and recommendations for at-risk patients, carry out standing orders with nursing staff, and develop protocols and collaborative agreements with physicians. ${ }^{22,37-39}$ Guidelines on the pharmacists' role in immunization in the inpatient or health-systems setting have been published by the American Society of Health-System Pharmacists (http://www.ashp.org/s ashp/ docs/files/BP07/Specific_Gdl_Immun.pdf [accessed May 14, 2015]). ${ }^{40}$

The remainder of this review highlights the roles and contributions of community pharmacists as vaccine providers through the roles of educators, facilitators, and immunizers.

\section{Pharmacists as vaccine educators}

Community pharmacists are valuable sources of information for patients. As vaccine educators, pharmacists act to educate and recommend to the patients the importance of and need for receiving vaccinations. Physician views toward the community pharmacist's role in patient advocacy include assisting physicians in monitoring pharmacotherapy, and providing patient counseling and medical information. ${ }^{30,41}$ The coordination and education regarding the importance of receiving routine and recommended vaccinations, and the vaccine product itself, would fall into this view of community pharmacists as sources of information. As discussed earlier, pharmacists have been trained in providing clinical services and patient communication; it is only appropriate that they employ this training in advocating vaccinations. Pharmacist-provided patient vaccine education, screening, and recommendations have been shown to increase vaccination rates. $^{42}$

Pharmacists have been successful in their role as vaccine educators by screening patients and providing recommendations to patients and providers. As providers of medication therapy management and a source of patient medication records, community pharmacists are able to identify patients at risk for vaccine-preventable diseases through use of pharmacy data and patient interviews. ${ }^{43,44}$ In an interview of seniors taking three or more medications concurrently according to pharmacy records, the most common pharmacist-identified drug-related problems were "patient required drug therapy" or "patient requires influenza or pneumococcal vaccination". ${ }^{43}$ Pharmacists are able to review prescription records to create "inferred diagnosis" when the medical record is not available, and identify the need for a specific vaccination. For example, screenings for the pneumococcal vaccine can target those 65 years of age or older, those with immunocompromising medications, those with respiratory or diabetes medications; or the Tdap (tetanus, diphtheria, and pertussis) vaccine for females with prenatal prescriptions or picking up infant-care supplies. This information can be obtained from the patient's pharmacy profile and prescription history in the pharmacy information management system (PIMS). The screening for and recommending of vaccinations are part of the pharmacist's responsibility in monitoring drug therapy, and includes identification of lack of therapy and preventative measures such as routine and recommended vaccinations.

Community pharmacists also educate the community through awareness campaigns and distributing literature on the need for vaccination and where to obtain the needed vaccinations. Using a combination of screening pharmacy records, distributing vaccine literature, and urging vaccination, community pharmacists in the Isle of Wight, England, vaccinated $9.7 \%$ of all patients who received influenza vaccine on the island during the 2010-2011 influenza season. They also noted that it was a pharmacy staff reminder that led to the initiation of two-thirds of these vaccinations. ${ }^{45}$ 
Similar results exist for pharmacist-driven interventions for the zoster vaccine. Pharmacists and pharmacy staff who promoted the zoster vaccine and provided personal selling and patient education were able to increase the number of zoster vaccinations compared with when there was no pharmacist intervention. ${ }^{46-49}$ With a lack of physician recommendation to patients and patients' misassumption of their perceived need for vaccinations, pharmacists can recapture these patients and engage them in conversation about needed vaccinations. ${ }^{50}$ The pharmacist's role as a vaccine educator enhances patient adherence to vaccinations through initiating an open discussion, providing education, and patient follow-up with the pharmacist. These techniques are similar to those employed in the pharmacist's role in improving patient adherence to other medications. ${ }^{51}$

\section{Pharmacists as vaccine facilitators}

As discussed earlier, early pharmacist involvement with immunizations was limited to the distribution of vaccine products and hosting of immunization providers in their pharmacy. Community pharmacists facilitated immunizations given by other health care providers, such as physicians and nurses, by providing their pharmacies as venues to provide vaccines. Hosting other providers was usually limited to 2-3 days during the fall and for a short number of hours during each event. Revenue generated from such events was also retained by the providers of immunization, and the pharmacy benefited through goodwill and collateral sales. ${ }^{52}$ However, with all states currently allowing pharmacists to immunize, modern community pharmacists now use their pharmacies to host their own immunization services year-round. This movement away from being distributors or facilitators to being full providers of immunizations may explain the scarce literature on the role of pharmacists as vaccine facilitators and distributors.

As vaccine distributors, pharmacies facilitate other providers in administering vaccinations by ordering and distributing vaccine products to physicians and medical clinics. In a random sample of community pharmacies from 17 states, about one in five pharmacies engaged in vaccine distribution by reselling or distributing vaccines to local physicians and/ or clinics. ${ }^{53}$ Common vaccines that were distributed to other providers for administration were the hepatitis $\mathrm{A}$, hepatitis $\mathrm{B}$, meningococcal, MMR (measles-mumps-rubella), and tetanus-containing vaccines. ${ }^{54}$ Although this is a minor role for pharmacy, it should be continued. A survey of family practitioners and general internists revealed that many primary care providers (PCPs) do not stock all recommended adult vaccines, with only $12 \%$ planning to increase their offerings and $2 \%$ planning to stop altogether. ${ }^{55}$ With barriers in reimbursements for pharmacy-administered vaccinations, some patients insist on receiving vaccinations from their PCP because it is covered under their medical benefits. In this case, PCPs will need access to vaccines they do not normally stock.

The pharmacist's role as a facilitator improves immunization rates by increasing other health care providers' accessibility to vaccine products and the locations where these providers can offer immunization services. In this role, pharmacists also aid other providers in improving their immunization offerings and rates of immunizations. It is important to note that while community pharmacists no longer serve in the originally defined role of facilitators (hosting other providers of immunizations), serving as facilitators was important in the progression of community pharmacists to immunizers by presenting the public with the concept of vaccination delivery in the pharmacy setting. For countries looking to implement pharmacy-based immunization delivery services, we suggest pharmacies serve as vaccine facilitators to trial immunization services in the pharmacy and expose the public and health system to vaccine delivery occurring in the community pharmacy.

\section{Pharmacists as immunizers}

According to the APhA Annual Pharmacy-Based Influenza and Adult Immunization Survey 2013, pharmacists provide vaccinations in $86 \%$ of community pharmacy settings. Patients are also increasingly being referred to the pharmacy from their PCP for immunizations by the pharmacists. ${ }^{56}$ With pharmacists authorized to administer vaccines in all 50 states, the most effective and efficient pharmacist role for providing vaccination services is to serve as an immunizer. As active immunizers, pharmacists assess patients for indications and contraindications and administer vaccines directly to the patients that they serve. Immunizing pharmacists follow the recommendations and immunization schedules provided by the Advisory Committee on Immunization Practices and the CDC. In a review of interventions to increase influenza and pneumococcal vaccination rates among community-dwelling adults, results showed that pharmacist interventions were ineffective when pharmacists only gave reminders to physicians and did not themselves administer the vaccinations. ${ }^{57}$ This role as an immunizer offers pharmacists the ability to deliver complete and successful immunization services by combining the roles of vaccine educator and immunizer. 
The literature is abundant with data supporting the role and impact of community pharmacists as immunizers. Community pharmacy-based immunization services are a cost-effective, convenient, and accessible alternative for the public to receive vaccinations. ${ }^{10,15}$ As stated earlier, one of the greatest barriers to vaccinations is accessibility. With pharmacists as immunizers, pharmacists are able to immediately act on their recommendations (administer the patient the vaccine) without referring the patient elsewhere, where the patient may not follow through or forget. With increased accessibility, pharmacists have helped to improve immunization rates, bring patients up-to-date on vaccinations, and reach those who may not otherwise have an opportunity to be vaccinated. ${ }^{11,18,45,58-60}$ When comparing patients 50 years or older who obtained their medications from a community pharmacy to those that did not, community pharmacy patients had a higher rate of receiving the influenza immunization. ${ }^{61}$ Prior to all states allowing pharmacists to provide vaccinations, states that allowed pharmacists to vaccinate were associated with higher influenza vaccination rates for those 65 years and older. ${ }^{62}$ In emergency-response situations requiring mass vaccinations, pharmacist immunizers, with a higher patient-to-practitioner ratio, have also been able to reach a larger portion of the community than other types of vaccine providers. ${ }^{63}$

\section{Practice model}

An outsourced or in-house approach can be taken to implementing immunization services inside the community pharmacy. ${ }^{64}$ In the outsourced model, pharmacies may contract with other professionals, notably nurses, to provide immunization services within the pharmacy during certain months, for example, influenza season and back to school months. The outsourced approach is essentially pharmacies participating in the vaccine facilitator role. Using an outsourced mechanism does not require much commitment from the hosting pharmacy beyond providing space in the pharmacy for the contracted immunization provider to work. Outsourced immunization services also require minimal pharmacy staff participation, allow for the trial of immunization services within the pharmacy, and are less likely to disrupt dispensing activities. ${ }^{64}$ This model is no longer used in US community pharmacies, since most pharmacies have an immunization-trained pharmacist on staff to carry out the vaccine-administration services within their own pharmacy.

Community pharmacies tend to utilize their immunizationtrained staff pharmacists to deliver all aspects of the immunization service alongside their distribution services. This is the second approach to implement pharmacy-based immunization services and is known as an in-house vaccination service. Of the two mechanisms to deliver pharmacy-based immunization services, the in-house vaccination service offers the most effective and constructive outcome to public health and the pharmacy profession. As stated before, in-house services, where pharmacists are immunizers, improve vaccination rates and increase access/convenience for patients. $44,45,59,61$ In-house pharmacy immunization services have also been shown to be more cost-effective compared with physician office vaccinations..$^{10}$ Unlike the outsourced method, in-house services are able to provide a wider range of vaccines yearround in the pharmacy and allow the pharmacy to retain the revenue generated from the immunization services. ${ }^{52}$ These in-house services improve the pharmacy business by bringing in revenue; patient care, by increasing vaccine and vaccineprovider access for patients; and the pharmacy role/profession, by expanding the role of clinical pharmacy services. ${ }^{65}$ Some published guides are available to assist pharmacists in developing and implementing clinical pharmacy services in the community setting. ${ }^{66,67}$

Pharmacist-provided immunizations typically require a protocol with a physician, similar to the standing orders that nurses, nurse practitioners, and physician assistants may operate under. ${ }^{24}$ Depending on the specific state's laws and regulations, pharmacists may initiate and administer vaccines under a collaborative practice agreement, protocol, or prescription order. The source and authorization of pharmacists provided with immunization protocols or standing orders are driven by the individual state's laws and regulations. In general, protocols allow one physician to sign for multiple pharmacists and all patients who present to the pharmacy. While the protocol is currently the most common authorization for pharmacists to immunize, it can also serve to limit immunization access. Protocols cannot be more liberal than a state law, but they can be stricter. For example, physicians or organizations may, for example, raise the age limit; prohibit the vaccination of pregnant women; or require a prescription for pediatric vaccines, even though state law does not require it. Lastly, it may be difficult for independent pharmacists to find a physician to sign their protocol because the physician may perceive there to be greater liability than there really is or lack of a financial incentive. Chain pharmacies often either employ their protocol physician or are able to provide enhanced liability protection and financial incentives to a protocol physician.

The delivery and workflow of an in-house community pharmacy immunization service varies from pharmacy 
to pharmacy. Immunization services can be provided by appointment, on a walk-in basis, or a combination of both. The Annual Pharmacy-Based Influenza and Adult Immunization Survey 2013 reported that a majority of pharmacy practice sites offer vaccinations on a walk-in basis, with an increasing trend for pharmacies to adopt the walk-in service. ${ }^{56}$ Appointment-based services tend to be less disruptive to the pharmacy's normal workflow, as patient volume is planned and expected, however it can limit the number of patients who can be vaccinated. Walk-in-based services are more convenient for patients but may cause disturbances to the normal pharmacy workflow to accommodate the patients. Walk-in services might also mean that the pharmacy has to stock a wide range of, if not all, vaccines at all times because the requested vaccine and patient screening cannot be determined in advance. The time it takes to provide the comprehensive vaccination service can vary depending on the number of pharmacists or student pharmacists available; pharmacy dispensing volume; insurance billing versus paying out-of-pocket; and patient screening, counseling, and education needs. The actual time it takes to administer the vaccine may only take 5 minutes or less. ${ }^{68}$

Other essential workflow and physical pharmacy environment needs for an in-house immunization service are a private patient-care area, adequate vaccine storage equipment, and emergency-response preparedness. While most pharmacies have been designed without private patient exam rooms, offering an immunization service only requires a semiprivate patient-care area. Pharmacies offering immunizations and patient-care services have met this need by repurposing existing space, or some have required remodeling of the physical pharmacy floor plans. A pharmacy providing vaccination services is also required to have adequate refrigeration and freezer equipment to ensure the potency and safe storage of vaccine products. Pharmacists who provide immunizations should also be adequately prepared for the management of adverse reactions that may occur. These may range from injection-site reactions and syncope to more severe reactions such as anaphylaxis. In addition to clear written protocols on the management of these reactions, immunizing pharmacists should be trained in cardiopulmonary resuscitation and have emergency supplies that consist of epinephrine, diphenhydramine, a blood pressure cuff, and a stethoscope. Upon completion of the injection, patients are asked to remain seated in the pharmacy for observation for approximately 15 minutes for any immediate syncopal or anaphylactic reactions. The $\mathrm{CDC}$ has published general recommendations regarding communicating risk and benefits, administration and storage, management of adverse events, special populations, record keeping, and resources for clinicians involved in the delivery of immunization services. ${ }^{69}$ The National Vaccine Advisory Committee has also provided recommendations for the standards of adult immunization practices to improve immunization practice and policy development. ${ }^{70,71}$

\section{Current challenges}

Despite the successes, community pharmacy-based vaccination services still face some challenges. Barriers noted include variability in laws at the state level regarding pharmacists' administration of vaccines, inconsistent reimbursement and compensation systems, and barriers to accessing health information technology. Although pharmacists in all 50 states and US territories are authorized to administer vaccines, the process (allowed under protocol, standing order, and/or prescription), patient age, and type of vaccine permitted vary considerably. These scopes of practice are determined individually at the state level, and the variability and limitations of some state regulations restrain the ability of pharmacists to fully contribute to immunization public-health efforts. The lack of recognition by all states that pharmacists are appropriately trained and professionally competent providers of immunizations who are able to increase the rate of vaccinations and improve public health may explain why some states still place limits on pharmacist immunization authorities. Individual states, with their pharmacists and local pharmacy associations, should continue to work with their state pharmacy boards and other stakeholders to advance their immunization scope of practice. This lack of wide recognition is also said to be the reason why inconsistent reimbursement designs for pharmacy-provided immunizations exist. ${ }^{25}$

For community pharmacy-based vaccination services, a limited number of commercial and governmental health plans offer patients coverage for receiving vaccinations from a pharmacy, and, even when provided, they do not always cover all recommended vaccines. ${ }^{72}$ For example, Medicare covers the pneumococcal and influenza vaccine in the pharmacy under the Part B medical benefits, but the shingles vaccine is covered under the Part D prescription benefits. This may also be a barrier for patients looking to receive a set of vaccinations from their PCP, who cannot bill under prescription drug benefits. Generally, for commercial health plans, if a vaccine is not covered under the pharmacy benefits, it is covered under the patient's medical benefits, which the pharmacy may have difficulty billing. Limited or restricted benefits for pharmacy-provided immunizations mean that the patient either has to go elsewhere or pay high 
out-of-pocket fees. This may result in the patient skipping the vaccine altogether or deferring vaccination. This is further complicated by the fact that most patients like the convenience of going to a pharmacy versus making an appointment with their PCP, and that many may seek vaccinations after normal physician office hours. ${ }^{18}$ Immunization services carry a cost for the vaccine product itself and for the cost of the administration. For physicians and for pharmacists, many times, the reimbursements barely cover the cost of the vaccine product and administration, resulting in no net revenue or even a loss. ${ }^{73,74}$ Immunization stakeholders believe that if all CDC-recommended vaccines can be billed from either the pharmacy or medical benefit (with uniformity in cost sharing and reimbursement for product and administration cost across all provider types), it will decrease the barriers to patient access to immunizations and incentivize pharmacies (and other immunizers) to increase their immunization offerings and efforts. ${ }^{24}$ As medical care efforts increasingly focus on preventative measures in accordance with the Affordable Care Act (2010), there are increased incentives for benefit designs to include pharmacy-based vaccination benefits. $^{72}$ Pharmacists are also encouraged to work with private employers/employer plans to seek further avenues for reimbursements and reaching more of the public.

There are also barriers in technology that exist in this setting; from a lack of shared patient record systems to inadequate PIMSs that provide patient-specific immunization notifications and scheduling of patients. Currently, only about $63 \%$ of pharmacy immunization sites surveyed provide documentation of patients' immunization directly to the PCP, and only $35 \%$ report to an immunization registry (with another $11 \%$ stating pharmacists are not permitted access to their state or local immunization registries). ${ }^{56} \mathrm{~A}$ lack of patient record systems shared with the patient centered medical home (PCMH), the PCP charged with coordinating care, has led to inconsistent communication between pharmacists and PCPs regarding patients' current immunization status and records. Also, variability exists in state-level laws regarding mandates for pharmacists and other immunization providers to report to the state's immunization information system (IIS) or immunization registries. ${ }^{75}$ The IIS gathers data on patients' immunization records from a variety of immunization providers in the hope of compiling a complete record for continuity of care and public health. Immunization registries in which not all providers and all vaccines are mandated to be entered provide an incomplete record of an individual's immunization status, possibly leading to under- or over-vaccination. Some challenges conveyed for pharmacy IIS reporting are that many PIMSs cannot directly communicate with the IIS; time-consuming paperwork and disruption of workflow; and lack of standard reporting mandates, procedures, and formats. ${ }^{76}$ Some health information technology companies have been working on solutions to overcome some of these issues, however, "the capabilities for pharmacies to interface with [IIS] at this level are still in the early stages". ${ }^{75}$ Another limitation in technology that may create some challenges for community pharmacy-based immunization services is that PIMSs used by pharmacies are generally not equipped with patient scheduling and patient recommendation features based on the patient's records. These features assist in patient follow-up and scheduling of vaccines that consist of multiple-dose series; they also help assist the pharmacist in prompting recommendations to the patients after the technology has queried the patient's records for indications that may correlate with age, medications, and/ or diagnoses. These electronic clinical reminders are typically seen in electronic medical records and not in a PIMS.

\section{Future considerations}

Looking into the future, there are actions that can be taken to ensure the continual success and expansion of community pharmacy-based vaccination services. To do this, we should focus future efforts on education, national legislative changes, reimbursement design, integration of immunization workflow into a PIMS, and communication with the PCMH. As health care needs and public-health initiatives grow and change, the training and education of student and current pharmacists must also be revised to meet these changes effectively. An increased emphasis on the role of pharmacists in public health will help pharmacists increase their credibility, expand their scope of practice at the micro- and macro-levels of public health, and hopefully provide pharmacist recognition and legislative changes at a national level as providers that are part of the PCMH. ${ }^{77-79}$ Ways to achieve this may be to incorporate public-health courses and public-health experiential education opportunities into the Pharm D curriculum, offer joint Pharm D and Master of Public Health (MPH) degree programs, increase continuing education topics in public health, and have pharmacists and pharmacy stakeholders participate in legislative actions. It has been suggested that pharmacists functioning as vaccine educators and immunizers could increase educational campaigns focusing on disparities and aimed at bridging the gap among racial and ethnic populations. ${ }^{61}$ Pharmacists can also expand beyond the community pharmacy to other community settings (churches, malls, senior centers, colleges, workplaces, etc) and have these locations host an immunizing pharmacist. 
Legislative and regulatory changes that will benefit the future sustainability of community pharmacy-based vaccination are standardized recognition for pharmacists to furnish all CDC-recommended vaccines to indicated patients as well as uniform provider recognition among benefit plans for improved reimbursement and compensation. Other avenues for increasing performance may be to implement vaccine-related performance measures or incentives in immunizing pharmacists' payer contracts. Although no states permit pharmacy technicians to administer vaccines, the pharmacy technician can serve a role in the pharmacy's immunization services. Pharmacy technicians can be delegated with tasks related to inventory, patient scheduling, and intake; prescription processing and payment; and administrative work. Engaging the pharmacy technician can minimize the time pharmacists spend on these areas and cause less disruption in the pharmacist's normal workflow. Another advancement that can assist in improving the pharmacists' efforts and save time is the use of PIMS software that can provide electronic clinical alerts for patient immunization needs, send reports to the state IIS, and communicate with PCPs.

\section{Conclusion}

With the appropriate training/education, accessibility, and effective provision of immunizations in community pharmacies, pharmacists are uniquely positioned to improve immunization rates and public-health efforts. Serving as immunization advocates in the roles of educators, facilitators, and immunizers, community pharmacists motivate patients to receive immunizations, enable other providers to immunize, and directly vaccinate the patients that they serve. Although much success has been seen in the past few decades, as evidenced by all pharmacists now being authorized to immunize, barriers such as variable scope of immunization practice for pharmacists across states, inconsistent reimbursement mechanisms, and barriers in technology still exist in this setting. Continued and future success in this area requires calls for action from educators, legislators, payers, and the health information technology community.

\section{Disclosure}

Albert Bach has no conflicts of interest to disclose in this work. Jeff Goad is on the Merck Vaccine speakers' bureau.

\section{References}

1. Levi J, Schaffner W, Cimons M, Guidos R, Segal LM; Trust for America's Health, Infectious Disease Society of America (IDSA), Robert Wood Johnson Foundation. Adult Immunization: Shots to Save Lives. Issue brief. Washington DC: Trust for America's Health; 2010. Available from: http://healthyamericans.org/assets/files/TFAH2010AdultImmnzBrief13. pdf. Accessed May 13, 2015.
2. US Department of Health and Human Services (DHHS) National Vaccine Advisory Committee (NVAC). The National Vaccine Advisory Committee (NVAC): the action plan [web page on the Internet]. Washington DC: DHHS; nd. Available from: http://www.hhs.gov/nvpo/ nvac/adult4.html. Accessed May 13, 2015.

3. Williams WW, Lu PJ, O'Halloran A, et al; Centers for Disease Control and Prevention (CDC). Noninfluenza vaccination coverage among adults - United States, 2012. MMWR Morb Mortal Wkly Rep. 2014;63(5):95-102.

4. HealthyPeople.gov. Immunization and infectious diseases: overview [web page on the Internet]. Washington DC: DHHS; nd. Available from: http://www.healthypeople.gov/2020/topicsobjectives2020/overview. aspx?topicid=23. Accessed September 16, 2014.

5. CDC. Flu Vaccination Coverage: United States, 2012-2013 Influenza Season. Atlanta, GA: CDC; nd [updated September 25, 2013]. Available from: http://www.cdc.gov/flu/pdf/fluvaxview/vax-coverage-1213estimates.pdf. Accessed May 13, 2015.

6. Hales CM, Harpaz R, Ortega-Sanchez I, Bialek SR; Centers for Disease Control and Prevention (CDC). Update on recommendations for use of herpes zoster vaccine. MMWR Morb Mortal Wkly Rep. 2014:22;63(33):729-731.

7. NACDS 2010-2011 Chain Pharmacy Industry Profile illustrates pharmacy value. Drug Topics [serial online]. September 21, 2010. Available from: http://drugtopics.modernmedicine.com/drug-topics/ news/modernmedicine/modern-medicine-news/nacds-2010-2011chain-pharmacy-industry-profile?page=full. Accessed May 13, 2015.

8. CDC. Place of influenza vaccination among adults - United States, 2010-2011 influenza season. MMWR Morb Mortal Wkly Rep. 2011;60(23):781-785.

9. Lu PJ, O'Halloran A, Ding H, Williams WW, Bridges CB, Kennedy ED. National and state-specific estimates of place of influenza vaccination among adult populations - United States, 2011-2012 influenza season. Vaccine. 2014;32(26):3198-3204.

10. Prosser LA, O'Brien MA, Molinari NA, et al. Non-traditional settings for influenza vaccination of adults: costs and cost effectiveness. Pharmacoeconomics. 2008;26(2):163-178.

11. Murphy PA, Frazee SG, Cantlin JP, Cohen E, Rosan JR, Harshburger DE. Pharmacy provision of influenza vaccinations in medically underserved communities. J Am Pharm Assoc (2003). 2012;52(1):67-70.

12. Postema AS, Breiman RF; NVAC. Adult immunization programs in nontraditional settings: quality standards and guidance for program evaluation. MMWR Recomm Rep. 2000;49(RR-1):1-13.

13. US Department of Labor Bureau of Labor Statistics. Pharmacists. In: Occupational Outlook Handbook [online]. 2014-2015 ed. Washington DC: US Department of Labor Bureau of Labor Statistics; 2014. Available from: http://www.bls.gov/ooh/healthcare/pharmacists.htm. Accessed May 13, 2015.

14. National Community Pharmacists Association (NCPA). 2010 NCPA Digest. Alexandria, VA: NCPA; 2010.

15. Grabenstein JD, Guess HA, Hartzema AG, Koch GG, Konrad TR. Attitudinal factors among adult prescription recipients associated with choice of where to be vaccinated. J Clin Epidemiol. 2002;55(3): 279-284.

16. Anderson C, Thornley T. "It's easier in pharmacy": why some patients prefer to pay for flu jabs rather than use the National Health Service. BMC Health Serv Res. 2014;14:35.

17. Ernst ME, Bergus GR, Sorofman BA. Patients' acceptance of traditional and nontraditional immunization providers. JAm Pharm Assoc (Wash). 2001;41(1):53-59.

18. Goad JA, Taitel MS, Fensterheim LE, Cannon AE. Vaccinations administered during off-clinic hours at a national community pharmacy: implications for increasing patient access and convenience. Ann Fam Med. 2013;11(5):429-436.

19. Crawford ND, Blaney S, Amesty S, et al. Individual- and neighborhoodlevel characteristics associated with support of in-pharmacy vaccination among ESAP-registered pharmacies: pharmacists' role in reducing racial/ethnic disparities in influenza vaccinations in New York City. J Urban Health. 2011;88(1):176-185. 
20. Accreditation Council for Pharmacy Education (ACPE). Accreditation Standards and Guidelines for the Professional Program in Pharmacy Leading to the Doctor of Pharmacy Degree. Chicago, IL: ACPE; 2006. Available from: https://www.acpe-accredit.org/pdf/ FinalS2007Guidelines2.0.pdf. Accessed May 13, 2015.

21. ACPE. Accreditation Standards and Key Elements for the Professional Program in Pharmacy Leading to the Doctor of Pharmacy Degree: Standards 2016. Chicago, IL: ACPE; 2014. Available from: https://www. acpe-accredit.org/pdf/Standards2016FINAL.pdf. Accessed March 27, 2015.

22. Hogue MD, Grabenstein JD, Foster SL, Rothholz MC. Pharmacist involvement with immunizations: a decade of professional advancement. J Am Pharm Assoc (2003). 2006;46(2):168-179; quiz 179-182.

23. American Pharmacists Association (APhA). Pharmacy-based immunization delivery [program description; web page on the Internet]. Washington DC: APhA; nd. Available from: http://www.pharmacist.com/pharmacybased-immunization-delivery. Accessed May 13, 2015.

24. Skelton JB; American Pharmacists Association, Academy of Managed Care Pharmacy. Pharmacist-provided immunization compensation and recognition: white paper summarizing APhA/AMCP stakeholder meeting. J Am Pharm Assoc (2003). 2011;51(6):704-712.

25. Goad J, Durham M. The anti-vaccine movement: a pharmacist's view. In: Chatterjee A, editor. Vaccinophobia and Vaccine Controversies of the 21st Century. New York, NY: Springer; 2013:119-128.

26. Gallup. Honesty/Ethics in professions [web page on the Internet]. Washington DC: Gallup; nd. Available from: http://www.gallup.com/ poll/1654/honesty-ethics-professions.aspx. Accessed May 13, 2015.

27. Grabenstein JD. Pharmacists and immunization: increasing involvement over a century. Pharm Hist. 1999;41(4):137-152.

28. Keely JL; American College of Physicians-American Society of Internal Medicine. Pharmacist scope of practice. Ann Intern Med. 2002;136(1):79-85.

29. NVAC. Recommendations from the National Vaccine Advisory Committee: standards for adult immunization practice. Public Health Rep. 2014;129(2):115-123.

30. Bradshaw SJ, Doucette WR. Community pharmacists as patient advocates: physician attitudes. J Am Pharm Assoc (Wash). 1998;38(5): 598-602.

31. American Academy of Family Physicians (AAFP). Pharmacists (position paper) [web page on the Internet]. Leawood, KS: AAFP; nd. Available from: http://www.aafp.org/about/policies/all/pharmacists.html. Accessed May 13, 2015.

32. Thomas J. Re: Senate Bill 493 (Hernandez). 2013. Available from: http://www.cshp.org/sites/main/files/cma_sb_493_hernandez_neutral. pdf from http://www.cshp.org/sb-493. Accessed September 16, 2014.

33. Barcellona WJ. Re: support - SB 493 (Hernandez) pharmacists [letter]. July 30, 2013. Available from: http://www.cshp.org/sites/main/files/ capg_sb_493_support_1tr._7_30_13.pdf. Accessed May 13, 2015.

34. APhA. Pharmacist Administered Vaccines [slide show]. Washington DC: APhA; nd [updated January 31, 2015]. http://www.pharmacist. com/sites/default/files/files/Pharmacist_IZ_Authority_1_31_15.pdf. Accessed March 2, 2015.

35. Pharmacy practice. Hernandez E, trans 2013-2014. Available from: http://leginfo.legislature.ca.gov/faces/billTextClient.xhtml?bill_ id=201320140SB493. Accessed September 12, 2014.

36. APhA. Guidelines for pharmacy-based immunization advocacy [web page on the Internet]. Washington DC: APhA; nd [approved 1997]. Available from: http://www.pharmacist.com/guidelines-pharmacybased-immunization-advocacy. Accessed May 13, 2015.

37. Robke JT, Woods M. A decade of experience with an inpatient pneumococcal vaccination program. Am J Health Syst Pharm. 2010;67(2): $148-152$.

38. Skledar SJ, McKaveney TP, Sokos DR, et al. Role of student pharmacist interns in hospital-based standing orders pneumococcal vaccination program. J Am Pharm Assoc (2003). 2007;47(3):404-409.
39. Sokos DR. Pharmacists' role in increasing pneumococcal and influenza vaccination. Am J Health Syst Pharm. 2005;62(4):367-377.

40. American Society of Health System Pharmacists Council on Professional Affairs. ASHP guidelines on the pharmacist's role in immunization. Am $J$ Health Syst Pharm. 2003;60(13):1371-1377.

41. Owens C, Baergen R, Cady P. Multistate survey of primary care physician and midlevel provider attitudes toward community pharmacists. J Am Pharm Assoc (2003). 2009;49(4):538-543; quiz 544-545.

42. Fuchs J. The provision of pharmaceutical advice improves patient vaccination status. Pharm Pract (Granada). 2006;4(4):163-167.

43. Kassam R, Farris KB, Burback L, Volume CI, Cox CE, Cave A. Pharmaceutical care research and education project: pharmacists' interventions. J Am Pharm Assoc (Wash). 2001;41(3):401-410.

44. Grabenstein JD, Guess HA, Hartzema AG, Koch GG, Konrad TR. Effect of vaccination by community pharmacists among adult prescription recipients. Med Care. 2001;39(4):340-348.

45. Warner JG, Portlock J, Smith J, Rutter P. Increasing seasonal influenza vaccination uptake using community pharmacies: experience from the Isle of Wight, England. Int J Pharm Pract. 2013;21(6): 362-367.

46. Bryan AR, Liu Y, Kuehl PG. Advocating zoster vaccination in a community pharmacy through use of personal selling. J Am Pharm Assoc (2003). 2013;53(1):70-77.

47. Teeter BS, Garza KB, Stevenson TL, Williamson MA, Zeek ML, Westrick SC. Factors associated with herpes zoster vaccination status and acceptance of vaccine recommendation in community pharmacies. Vaccine. 2014;32(43):5749-5754.

48. Wang J, Ford LJ, Wingate L, et al. Effect of pharmacist intervention on herpes zoster vaccination in community pharmacies. $J$ Am Pharm Assoc (2003). 2013;53(1):46-53.

49. Hess R. Impact of automated telephone messaging on zoster vaccination rates in community pharmacies. JAm Pharm Assoc (2003). 2013;53(2): $182-187$.

50. Johnson DR, Nichol KL, Lipczynski K. Barriers to adult immunization. Am J Med. 2008;121(7 Suppl 2):S28-S35.

51. Bubalo J, Clark RK Jr, Jiing SS, et al. Medication adherence: pharmacist perspective. J Am Pharm Assoc (2003). 2010;50(3):394-406.

52. Grabenstein JD. Pharmacists as vaccine advocates: roles in community pharmacies, nursing homes, and hospitals. Vaccine. 1998;16(18): 1705-1710.

53. Hung SY, Watcharadamrongkun S, Kim M, Westrick SC, Mount JK. Involvement of community pharmacies in immunization promotion and vaccine distribution. Paper presented at The 41 st National Immunization Conference, Kansas City, MO, USA, March 5-8, 2007. Available from: https://cdc.confex.com/cdc/nic2007/techprogram/P12716.HTM. Accessed May 13, 2015.

54. Westrick SC, Watcharadamrongkun S, Mount JK, Breland ML. Community pharmacy involvement in vaccine distribution and administration. Vaccine. 2009;27(21):2858-2863.

55. Freed GL, Clark SJ, Cowan AE, Coleman MS. Primary care physician perspectives on providing adult vaccines. Vaccine. 2011;29(9): 1850-1854.

56. APhA. Annual Pharmacy-Based Influenza and Adult Immunization Survey 2013. Final Report. Washington DC: APhA; 2013. Available from: https://www.pharmacist.com/sites/default/files/files/Annual\%20 Immunization\%20Survey\%20Report.pdf. Accessed May 13, 2015.

57. Lau D, Hu J, Majumdar SR, Storie DA, Rees SE, Johnson JA. Interventions to improve influenza and pneumococcal vaccination rates among community-dwelling adults: a systematic review and metaanalysis. Ann Fam Med. 2012;10(6):538-546.

58. Papastergiou J, Folkins C, Li W, Zervas J. Community pharmacistadministered influenza immunization improves patient access to vaccination. Can Pharm J (Ott). 2014;147(6):359-365.

59. Higginbotham S, Stewart A, Pfalzgraf A. Impact of a pharmacist immunizer on adult immunization rates. $J$ Am Pharm Assoc (2003). 2012;52(3):367-371.

60. Taitel M, Cohen E, Duncan I, Pegus C. Pharmacists as providers: targeting pneumococcal vaccinations to high risk populations. Vaccine. 2011;29(45):8073-8076. 
61. Wang J, Munshi KD, Hong SH. Racial and ethnic disparities in influenza vaccinations among community pharmacy patients and non-community pharmacy respondents. Res Social Adm Pharm. 2014;10(1):126-140.

62. Steyer TE, Ragucci KR, Pearson WS, Mainous AG 3rd. The role of pharmacists in the delivery of influenza vaccinations. Vaccine. 2004;22(8):1001-1006.

63. Seib K, Gleason C, Richards JL, et al. Partners in immunization: 2010 survey examining differences among H1N1 vaccine providers in Washington state. Public Health Rep. 2013;128(3):198-211.

64. Westrick SC. Forward and backward transitions in pharmacy-based immunization services. Res Social Adm Pharm. 2010;6(1):18-31.

65. Westrick SC. Pharmacy characteristics, vaccination service characteristics, and service expansion: an analysis of sustainers and new adopters. J Am Pharm Assoc (2003). 2010;50(1):52-61.

66. Snella KA, Sachdev GP. A primer for developing pharmacist-managed clinics in the outpatient setting. Pharmacotherapy. 2003;23(9): 1153-1166.

67. American College of Clinical Pharmacy, Harris IM, Baker E, et al. Developing a business-practice model for pharmacy services in ambulatory settings. Pharmacotherapy. 2008;28(2):285.

68. Ernst ME, Chalstrom CV, Currie JD, Sorofman B. Implementation of a community pharmacy-based influenza vaccination program. $\mathrm{J} \mathrm{Am}$ Pharm Assoc (Wash). 1997;NS37(5):570-580.

69. National Center for Immunization and Respiratory Diseases. General recommendations on immunization - recommendations of the Advisory Committee on Immunization Practices (ACIP). MMWR Recomm Rep. 2011;60(2):1-64.

70. NVAC. A pathway to leadership for adult immunization: recommendations of the National Vaccine Advisory Committee: approved by the National Vaccine Advisory Committee on June 14, 2011. Public Health Rep. 2012;127 Suppl 1:1-42.

71. NVAC. Recommendations from the National Vaccine Advisory Committee: standards for Adult Immunization Practice. Public Health Rep. 2014;129(2):115-129.
72. Ko KJ, Wade RL, Yu HT, Miller RM, Sherman B, Goad J. Implementation of a pharmacy-based adult vaccine benefit: recommendations for a commercial health plan benefit. J Manag Care Spec Pharm. 2014;20(3):273-282.

73. Glazner JE, Steiner JF, Haas KJ, Renfrew B, Deutchman M, Berman S. Is reimbursement for childhood immunizations adequate? evidence from two rural areas in colorado. Public Health Rep. 2001;116(3): 219-225.

74. Institute of Medicine Committee on the Evaluation of Vaccine Purchase Financing in the United States Board on Health Care Services. Financing Vaccines in the 21st Century: Assuring Access and Availability. Washington DC: National Academies Press; 2003.

75. Bonner L. Pharmacists reporting to state immunization registries [web page on the Internet]. Washington DC: APhA; 2014. Available from http://www.pharmacist.com/pharmacists-reporting-state-immunizationregistries. Accessed December 19, 2014.

76. American Immunization Registry Association (AIRA). Survey of Immunization Reporting to Immunization Information Systems by Major US Pharmacies: A Summary of Methods, Successes, and Challenges of Pharmacy-IIS Interfaces. AIRA White Paper. Washington DC: AIRA; 2014. Available from: http://www.immregistries.org/resources/ aira_pharmcy_iis_white_paper.pdf. Accessed May 13, 2015.

77. Strand MA, Miller DR. Pharmacy and public health: a pathway forward. J Am Pharm Assoc (2003). 2014;54(2):193-197.

78. Truong HA, Patterson BY. Professional and educational initiatives, supports, and opportunities for advanced training in public health. Am J Pharm Educ. 2010;74(7):122.

79. American Public Health Association (APHA). The role of the pharmacist in public health [web page on the Internet]. Policy number 200614. Washington DC: APHA; 2006. Available from: http://www. apha.org/policies-and-advocacy/public-health-policy-statements/ policy-database/2014/07/07/13/05/the-role-of-the-pharmacist-inpublic-health\#Anchor\%201. Accessed May 13, 2015.

\section{Publish your work in this journal}

Integrated Pharmacy Research and Practice is an international, peer-reviewed, open access, online journal, publishing original research, reports, reviews and commentaries on all areas of academic and professional pharmacy practice. This journal aims to represent the academic output of pharmacists and pharmacy practice with particular focus on integrated care. All papers are carefully peer reviewed

\section{Dovepress}

to ensure the highest standards as well as ensuring that we are informing and stimulating pharmaceutical professionals. The manuscript management system is completely online and includes a very quick and fair peer-review system, which is all easy to use. Visit http://www.dovepress.com/testimonials.php to read real quotes from published authors. 